\title{
Anesthetic considerations of percutaneous transcatheter aortic valve implantation: first attempt in Korea -A report of 2 cases-
}

\author{
Hyo Jung Son, Hwa Mi Lee, Ji Hyun Chin, Dae Kee Choi, Eun Ho Lee, Ji Yeon Sim, and In Cheol Choi \\ Department of Anesthesiology and Pain Medicine, Asan Medical Center, University of Ulsan College of Medicine, Seoul, Korea
}

Conventional aortic valve replacement for severe aortic stenosis is associated with a high operative mortality in the elderly patients with significant comorbidities, including severe respiratory dysfunction, renal insufficiency, and compromised cardiac function. Human transcatheter aortic valve implantation was first reported in 2002 and has become a valid alternative in selected high-risk patients in Europe and North America. This article describes the first attempt of transfemoral transcatheter aortic valve implantation in Korea. The procedure was applied in two consecutive patients with severe aortic stenosis. Despite several intra-operative complications during procedure, the post-operative outcomes were good for both patients. At post-operative 30 days there was satisfactory prosthetic valve function and hemodynamic stability. (Korean J Anesthesiol 2011; 60: 128-133)

Key Words: Anesthesia, Aortic stenosis, Conventional aortic valve replacement, Percutaneous transcatheter aortic valve implantation.

Conventional aortic valve replacement (AVR) is the definitive treatment for severe aortic stenosis (AS). If untreated and symptoms of angina, syncope, or heart failure are allowed to develop, the average survival is 2 to 3 years, with a high risk of sudden death [1]. However, conventional AVR is associated with a high risk of operative mortality in elderly patients with significant comorbidities such as, severe respiratory dysfunction, renal insufficiency, and compromised cardiac function [2]. Therefore, the development of minimally invasive therapeutic options is comsidered important. The clinical use of transcatheter aortic valve implantation (TAVI) was first reported in 2002 [3]. TAVI is now considered a valid alternative to conventional surgery in selected high-risk patients in Europe and North America. The present report describes the first attempt of transfemoral TAVI in Korea and 30-day follow-up.

\section{Case Reports}

\section{Case 1}

An 83-year old woman with severe AS was scheduled for TAVI. She had had dyspnea on exertion (NYHA class III) for

Received: May 12, 2010. Revised: 1st, June 15, 2010; 2nd, August 20, 2010. Accepted: October 4, 2010.

Corresponding author: In Cheol Choi, M.D., Department of Anesthesiology and Pain Medicine, Asan Medical Center, University of Ulsan College of Medicine, 388-1, Pungnap 2-dong, Songpa-gu, Seoul 138-736, Korea. Tel: 82-2-3010-3862, Fax: 82-2-3010-6790, E-mail: icchoi@amc.seoul.kr (c) This is an open-access article distributed under the terms of the Creative Commons Attribution Non-Commercial License (http:// creativecommons.org/licenses/by-nc/3.0/), which permits unrestricted non-commercial use, distribution, and reproduction in any medium, provided the original work is properly cited. 
10 years, and this had aggravated recently. Her past history included hypertension, pulmonary tuberculosis when younger, and percutaneous coronary stent insertion in the left anterior descending artery (LAD), left circumflex artery (LCX), and right coronary artery (RCA) 5 months ago. She has taken aspirin $100 \mathrm{mg}$, clopidogrel $75 \mathrm{mg}$, and diltiazem $90 \mathrm{mg}$ daily, and all of these medications were continued. Transthoracic echocardiography showed severe AS with an aortic valve area (AVA) of $0.8 \mathrm{~cm}^{2}$, a mean pressure gradient of $41 \mathrm{mmHg}$ and an aortic annulus diameter of $21 \mathrm{~mm}$. Mild mitral regurgitation (MR) was present and the left ventricular ejection fraction was $62 \%$. Coronary angiography (CAG) showed that all stents were patent. Computed tomography (CT) showed diffuse calcification in the abdominal aorta and no tortuosity in either common iliac artery. The right iliac artery was $13.2 \mathrm{~mm}$ and the right femoral artery was $8.9 \mathrm{~mm}$ in diameter. The logistic EuroSCORE was $14.3 \%$.

The procedure was performed in the cardiac catheterization laboratory under general anesthesia. A 5-lead electrocardiograph, pulse oximeter, BIS sensor, and two cerebral oxymetry sensors were applied. Anesthesia was induced with etomidate $12 \mathrm{mg}$, rocuronium $50 \mathrm{mg}$, and continuous infusion of remifentanil (target effect site concentration $10 \mathrm{ng} / \mathrm{ml}$ ) and propofol (target effect site concentration $1.0 \mu \mathrm{g} / \mathrm{ml}$ ) using a target controlled infusion system (Orchestra ${ }^{\circledR}$, Fresenius vial, Brezims, France). An intra-arterial catheter was inserted into the left radial artery and endotracheal intubation was performed. A central venous catheter (MAC ${ }^{\mathrm{TM}}$ Two-Lumen Central Venous Access set, ARROW ${ }^{\circledR}$, Reading, PA, USA) with a Swan-Ganz catheter (Swan-Ganz CCOmbo, $\mathrm{CCO} / \mathrm{SvO}_{2} / \mathrm{CEDV}$, Edwards lifesciences LLC, Irvine, CA, USA) was inserted into the right internal jugular vein for continuous monitoring of cardiac output and mixed venous oxygen saturation using continuous cardiac output monitoring (Vigilance ${ }^{\circledR}$, Edwards lifesciences LLC, Irvine, CA, USA). During the procedure, anesthesia was maintained with oxygen ( $2 \mathrm{~L} / \mathrm{min})$, and continuous infusion of remifentanil (target effect site concentration 10-20 ng/ $\mathrm{ml}$ ), propofol (target effect site concentration 1.0-1.5 $\mu \mathrm{g} / \mathrm{ml}$ ) and rocuronium $0.5 \mu \mathrm{g} / \mathrm{ml} / \mathrm{min}$. Two external defibrillator pads were attached. Transesophageal echocardiography (TEE) (Live 3D TEE, iE33, Philips ultrasound, Bothell, WA, USA) was performed to confirm the aortic annulus diameter and to provide images for correct valve positioning during the procedure. Heparin $(1 \mathrm{mg} / \mathrm{kg})$ was given to achieve an activated coagulation time $>250$ seconds. The left femoral vein was used for the placement of a pacing wire (Electrophysiology catheter, St. Jude Medical ${ }^{\circledR}$, St. Paul, MN, USA) in the right ventricular apex and the left femoral artery was used for insertion of a pigtail catheter in the aortic root. Surgical exposure of the right femoral artery was performed and a 12 French introducer was inserted. Balloon valvuloplasty was successfully performed under rapid ventricular pacing (RVP) of 200 beats/min (Dual chamber temporary pacemaker, Medtronic $^{\circledR}$, Minneapolis, MN, USA) (Fig. 1). The duration of cardiac standstill during RVP was less than 10 seconds. Blood pressure and cardiac rhythm were restored well after RVP. Systolic blood pressure was 110$140 \mathrm{mmHg}$ and diastolic blood pressure was $50-60 \mathrm{mmHg}$ before and after RVP. The 12 French sheath was exchanged with a 24 French introducer sheath. Althought preoperative CT indicated that the right femoral artery was large enough for a 24 French introducer, it was a tight fit during the procedure, and insertion required the cardiologist to exert more force than anticipated. A $23 \mathrm{~mm}$ Edward-Sapien valve (Edwards Lifesciences LLC, Irvine, CA, USA) crimped onto a balloon was then advanced into the aortic annulus. The optimal prosthetic valve position was confirmed using TEE and fluoroscopy. The balloon was inflated after RVP. However, the prosthetic valve was ejected into the ascending aorta and moved down to the descending thoracic aorta. TEE was performed immediately and showed that the valve was packed in the descending thoracic aorta. However, there was no flow disturbance around the valve and the vital signs were stable. The cardiologist made a decision to leave the valve in place and insert another valve. The second valve crimped onto a balloon was advanced through the embolized valve. RVP was repeated several times to enable correct positioning and intermittent bolus injections of phenylephrine $(100 \mu \mathrm{g} / \mathrm{ml})$ were used without continuous infusion of a vasopressor. The second trial was successful. TEE

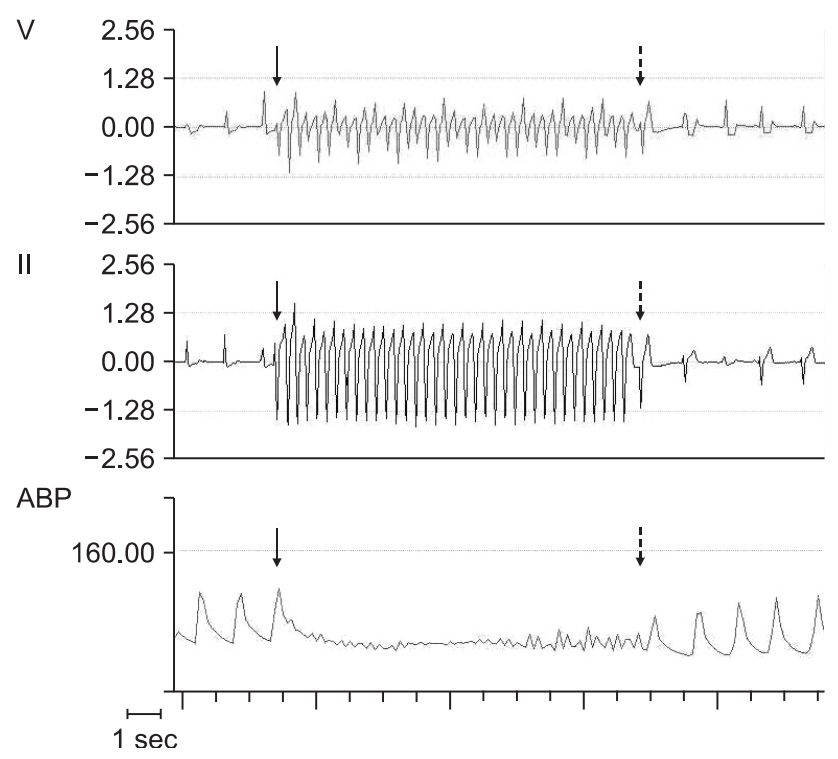

Fig. 1. Changes of electrocardiogram (lead V and lead II) and arterial blood pressure before and after rapid ventricular pacing. Arrow: commencement of rapid ventricular pacing, Dotted arrow: termination of rapid ventricular pacing. 
showed that the valve was stable and there was no paravalvular leakage. A root aortogram showed patent coronary arteries and no paravalvular leakage or aortic regurgitation (AR) (Fig. 2). After confirmation of valve function, the cardiac surgeon tried to remove the 24 French introducer, but initially found that it would not move in any direction. After approximately 30 minutes of manipulation, the surgeon was able to move the introducer backward. At that time, the mean arterial pressure abruptly decreased to $30 \mathrm{mmHg}$. Angiography was performed and showed a right external iliac artery rupture. Packed red blood cells and colloid solutions were rapidly infused. The cardiologist inserted the balloon catheter into the descending

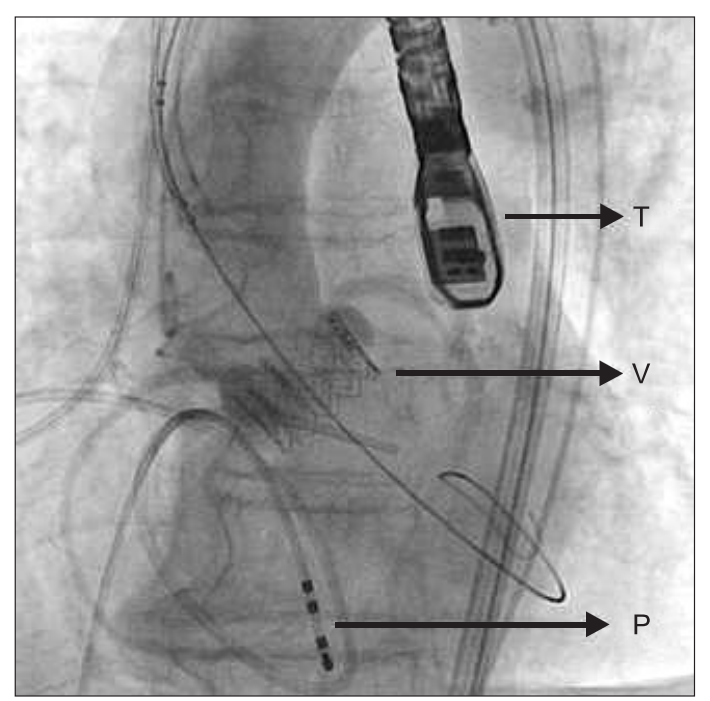

Fig. 2. Root aortogram after deployment of prosthetic aortic valve shows patent coronary arteries and no aortic regurgitation. T: transesopahgeal echocardiography probe, $\mathrm{V}$ : prosthetic aortic valve, P: pacemaker wire.

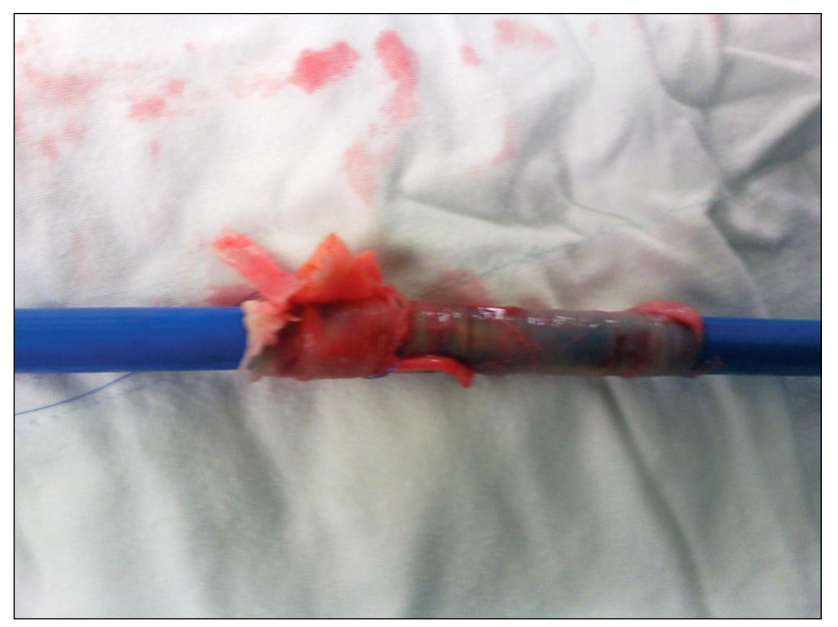

Fig. 3. The 24 French introducer sheath after removal. A segment of the external iliac artery is torn out with the sheath. aorta and inflated the balloon. A vascular surgeon performed an emergecy laparotomy and clamped the proximal side of the right external iliac artery. The patient was then sent to the operating room. The 24 French introducer was removed (Fig. 3) and an emergency femorofemoral bypass was performed. The total infused fluid volume was 14 units of packed red blood cells, 10 units of fresh frozen plasma, 10 units of platelets, 1,500 $\mathrm{ml}$ of cell saver blood, 4,400 ml of crystalloid solutions and 1,000 $\mathrm{ml}$ of colloid solutions. On post-operative day 1 , the patient was mentally alert, had good motor and sensory nerve function, and a normal pulse in her right lower extremity. Echocardiography showed a well-functioning prosthetic aortic valve, a mean pressure gradient of $16 \mathrm{mmHg}$, mild paravalvular leakage and mild AR. Chest radiography showed no migration of the embolized valve (Fig. 4). The patient was extubated on postoperative day 2 and sent to the general ward. The prosthetic aortic valve was functioning stable, however, the patient had ileus till post-operative day 30 .

\section{Case 2}

An 84-year old woman with severe AS was scheduled for TAVI. Her past history included hypertension, pulmonary tuberculosis when younger, and intermediate stenosis of LAD and LCX. She has taken aspirin $100 \mathrm{mg}$, isosorbide dinitrate $40 \mathrm{mg}$, and atenolol $50 \mathrm{mg}$ daily, and all of these medications were continued. Transthoracic echocardiography showed severe AS with an AVA of $0.8 \mathrm{~cm}^{2}$, a mean pressure gradient of $40 \mathrm{mmHg}$ and an aortic annulus diameter of $21.5 \mathrm{~mm}$. Mild AR and mild MR were present. The left ventricular ejection fraction was $66 \%$ and mild to moderate atherosclerosis was present in

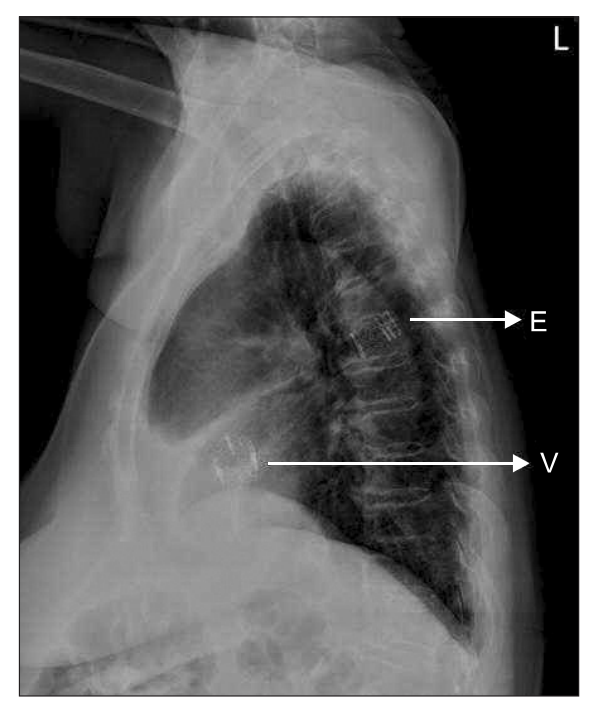

Fig. 4. Follow-up chest radiograph shows no movement of embolized valve. E: embolized prosthetic valve, V: prosthetic valve. 
the descending thoracic aorta and aortic arch. CAG showed $70-80 \%$ stenosis at the distal LAD and distal LCX. CT showed patent non-tortuous iliac and femoral arteries (the right iliac artery was $8.5 \mathrm{~mm}$ and right femoral artery was $7.3 \mathrm{~mm}$ in diameter). The logistic EuroSCORE was 6.14\%.

The anesthetic preparation was identical as that for case 1. Isosorbide dinitrate $1.0 \mu \mathrm{g} / \mathrm{kg} / \mathrm{min}$ was continuously infused after induction. Heparin $(1 \mu \mathrm{g} / \mathrm{kg})$ was given to achieve an activated coagulation time $>250$ seconds. Balloon valvuloplasty was successfully performed under RVP. Blood pressure and cardiac rhythm were restored well after RVP. Systolic blood pressure was 90-120 $\mathrm{mmHg}$ and diastolic blood pressure was 50-60 mmHg before and after RVP. However, ST segment elevation was observed in lead II and V, and worsened after repeated RVP. The infusion rate of isosorbide dinitrate was therefore increased and intermittent bolus injections of phenylephrine $(100 \mu \mathrm{g} / \mathrm{ml})$ were used. Prosthetic aortic valve implantation was successfully performed and ST segment elevation improved to the normal range. TEE showed a stable valve position and no paravalvular leakage (Fig. 5). A root aortogram showed patent coronary arteries and trace AR. After confirmation of valve function, a new-onset left bundle branch block was observed. The 24 French introducer was easily removed. The procedure took a total of 105 minutes. The patient was extubated uneventfully and sent to the ICU. Although she occasionally complained of chest discomfort without pain, electrocardiography and cardiac enzymes findings remained normal, and the symptoms resolved within 3 hours. The isosorbide dinitrate infusion was ceased, and she continued on her pre-operative oral medications with the addition of clopidogrel $75 \mathrm{mg}$. The patient was sent to the general ward on post-operative day 1 , and showed no signs of complications. Echocardiography showed a satisfactorily functioning

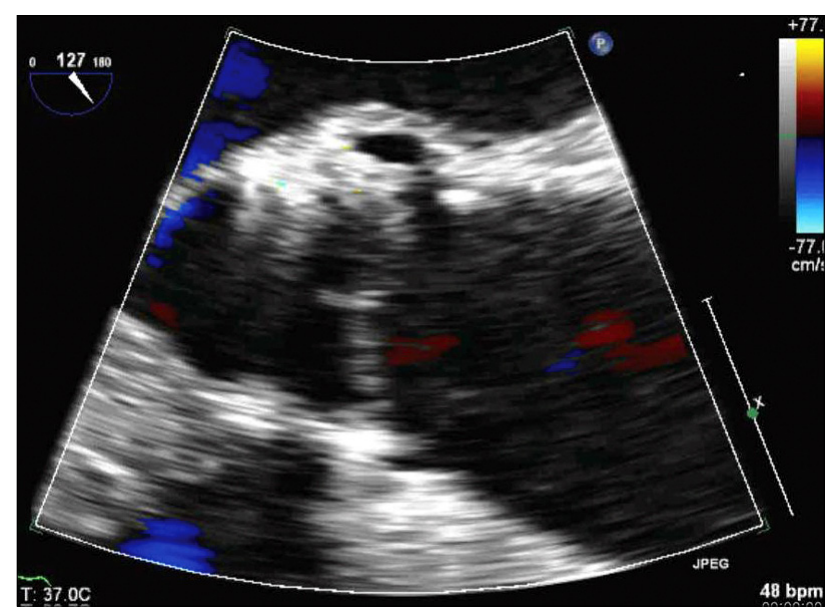

Fig. 5. Mid esophageal AV long axis view shows a stable valve position and no paravalvular leakage. prosthetic aortic valve, a mean pressure gradient of $21 \mathrm{mmHg}$ and mild AR. The patient was well at 30-day follow-up.

\section{Discussion}

Although conventional AVR is the gold standard treatment for symptomatic severe AS, and operative mortality is low in ideal candidates, its use is limited in the elderly with comorbidities [2]. Percutaneous balloon valvuloplasty was initially designed to treat valve stenosis less invasively. However, stenosis typically returns within 6 months following valvuloplasty [4]. Hence, TAVI was developed, and has been widely performed in selected high-risk patients, mainly in Europe and North America. The advantages of TAVI include avoidance of a sternotomy, cardiopulmonary bypass and cardiac arrest, along with a short hospital stay and fast return to functional status [5].

Recent National Institute for Health and Clinical Excellence (NICE) guidelines and licensing regulations have restricted the use of TAVI to selected patients not suitable for surgical AVR due to high operative mortality and risk of severe complications [6]. The EuroSCORE is usually used as a predictive risk model [2] and TAVI is recommended in patients with a logistic EuroSCORE over 20\%. Comprehensive baseline examination and careful patient selection is important to avoid complications at implantation. Patients underwent transthoracic echocardiography, coronary and aortofemoral angiography, and a multidetector CT to confirm adequate vascular dimensions and lack of tortuosity or intravascular thrombus. There are several contraindications of TAVI, which may change as the technology develops. These include bicuspid aortic valve (because the altered anatomy makes secure placement of the transcatheter valve which are potentially unstable), previous mechanical AVR, endocarditis, recent myocardial infarction or cerebrovascular accident, moderate to severe mitral or tricuspid valve regurgitation, left ventricular or atrial thrombus, an aortic annular diameter less than $18 \mathrm{~mm}$ or over $25 \mathrm{~mm}$, an ascending aorta diameter over $45 \mathrm{~mm}$, severe vascular calcification and aortic disease [7].

A hybrid operation room (a standard operation room with an additional angiography system equivalent to a standard catheterization laboratory) is recommended for the TAVI as a precaution for emergency such as device embolization, prolonged ventricular fibrillation, and massive hemorrhage. In our cases, the procedure was performed in the cardiac catheterization laboratory with operating room-like sterile precautions. A cardiac surgeon took active part in the procedure, and a vascular surgeon was on call and aware of the ongoing procedure. A perfusionist was present to institute emergency CPB. The anesthesiologist took a participatory role in monitoring and standards of care in the catheterization 
laboratory for this procedure.

General anesthesia or local anesthesia plus sedation can be used. Elective general anesthesia has many advantages, which includes facilitation of sheath placement, removal, and eventual surgical repair of the arterial access site, immobility during valve deployment, periprocedural TEE, airway management, minimal patient movement, avoidance of breathing artifacts, and rapid $\mathrm{CPB}$ institution when necessary [8]. Anesthetic preparation is identical to that for open cardiac surgery. Short-acting anesthetics are used to facilitate emergence and extubation at the end of the procedure.

The complication rates associated with TAVI have yet to be determined. Reported complications include poor recovery of cardiac function after RVP, paravalvular leakage, AR, myocardial ischemia and device embolization, complete heart block, newonset arrhythmia, neurological dysfunction or stroke, damage to the vascular access site, and radiocontrast induced acute renal failure [7]. RVP and cessation of mechanical ventilation interrupt cardiac ejection and minimize cardiac motion, facilitating exact prosthesis positioning within the native annulus. After termination of RVP, heart rate, rhythm, and arterial blood pressure usually return to baseline, but a bolus injection of a vasopressor or defibrillation may be required to restore stable hemodynamics. Both present patients showed stable hemodynamics after RVP. Intermittent bolus injections of phenylephrine $(100 \mu \mathrm{g} / \mathrm{ml})$ were used without continuous infusion of norepinephrine in both patients. Phenylephrine or norepinephrine is adequate for increasing systemic vascular resistance. Catecholamines such as epinephrine may worsen hypotension when administered to patients with left ventricular hypertrophy [9] and should be used with caution. The second present patient with intermediate coronary artery disease showed aggravation of ST segment elevation despite continuous isosorbide dinitrate infusion. That means deterioration of coronary artery flow after RVP. Therefore, it is necessary to consider the underlying coronary status and allow for sufficient rest periods of $1-2$ minutes after RVP. Paravalvular regurgitation is often present immediately after valve deployment, but improves after $24-48$ hours as valve morphology alters at body temperature [7]. Reinflation of the deployment balloon within the prosthesis or further expansion of the valve within the annulus is often employed for treatment of paravalvular AR. Significant AR suggests overexpansion of the prosthesis, which may require deployment of a second prosthetic valve within the first one [10]. Prosthetic valve embolization occurred in the first present case. We assumed it was the result of the insufficient cardiac standstill. To prevent this, a few seconds of delay for non-pulsatile flow through the aortic valve may be required. Previous reports documented that valve embolization has been experienced during initial attempts with the procedure [10].
Management of this complication requires repositioning of the prosthesis, implantation of an additional valve prosthesis, or an emergency sternotomy, CPB, aortic cross-clamping, and aortotomy to remove the embolized valve and perform open AVR [10]. Vascular injury at the access site is the most common complication and causes sudden and significant hemorrhage. Anesthesiologists should be alert to the possibility of a major vascular rupture, which requires urgent repair. To minimize the risk of this complication, careful evaluation of the arterial anatomy is necessary and the diameter of both the femoral and iliac arteries should be large enough for introducer sheath insertion. Also, those arteries should be free of severe calcification and not be tortous. In the first present case, althought the femoral and iliac arteries were considered large enough, sheath insertion was found to be difficult due to intraarterial calcification. We assumed that rupture of the common iliac artery occurred due to forceful insertion and removal of the sheath. The introducer sheath should be inserted and removed smoothly, and if it becomes stuck as our case, it should be removed in the operating room with preparation for emergency.

Systemic anticoagulation should be commence before TAVI and continue postoperatively. Usually, administering aspirin $300 \mathrm{mg}$ and clopidogrel $300 \mathrm{mg}$ before the procedure and starting regular dose of aspirin $100 \mathrm{mg}$ and clopidogrel $75 \mathrm{mg}$, soon after the end of the procedure, are recommended [8]. These are normally continued for 3-6 months. If a patient has been medicated as in our cases, loading dose of aspirin and clopidogrel are not necessary. Patients who re-present after TAVI for non-cardiac surgery can be managed similarly to patients after conventional AVR. There is no evidence that discontinuing anti-platelet drugs increases the risk of valve thrombosis perioperatively, and full heparinization is not required [7].

Although the procedure is still in its infancy and longterm outcomes are not yet known, the results from the first generation have been encouraging, demonstrating satisfactory technical feasibility, substantial reduction of the transaortic pressure gradient, and fast left ventricular function recovery [11]. Previous reports demonstrated that perioperative mortality and in-hospital mortality were $0-6 \%$ and $6.7-12 \%$, respectively, and that there was no evidence of structural device failure during 2 year follow-up [7,10,11].

In conclusion, TAVI has become an alternative to surgical AVR for the elderly with comorbidities who have a high operative mortality rate or are deemed non-operable. However, judicious selection of patients is important because TAVI can cause several serious complications. A multidisciplinary approach including a trained cardiac surgeon, interventional cardiologist, and cardiac anesthesiologist and a hybrid operation room is essential to provide safe and successful service. Anesthe- 
siologists must be aware of entire procedure and be prepared for sudden hemodynamic deterioration, which may require inotropic support, defibrillation, massive fluid infusion, or emergency surgery.

\section{References}

1. American College of Cardiology/American Heart Association Task Force on Practice Guidelines; Society of Cardiovascular Anesthesiologists; Society for Cardiovascular Angiography and Interventions; Society of Thoracic Surgeons, Bonow RO, Carabello BA, et al. ACC/AHA 2006 guidelines for the management of patients with valvular heart disease: a report of the American College of Cardiology/American Heart Association Task Force on Practice Guidelines (writing committee to revise the 1998 Guidelines for the Management of Patients With Valvular Heart Disease): developed in collaboration with the Society of Cardiovascular Anesthesiologists: endorsed by the Society for Cardiovascular Angiography and Interventions and the Society of Thoracic Surgeons. Circulation 2006; 114: e84-231.

2. Nashef SA, Roques F, Hammill BG, Peterson ED, Michel P, Grover FL, et al. Validation of European System for Cardiac Operative Risk Evaluation (EuroSCORE) in North American cardiac surgery. Eur J Cardiothorac Surg 2002; 22: 101-5.

3. Cribier A, Eltchaninoff H, Bash A, Borenstein N, Tron C, Bauer F, et al. Percutaneous transcatheter implantation of an aortic valve prosthesis for calcific aortic stenosis: first human case description. Circulation 2002; 106: 3006-8.

4. Otto CM, Mickel MC, Kennedy JW, Alderman EL, Bashore TM,
Block PC, et al. Three-year outcome after balloon aortic valvuloplasty. Insights into prognosis of valvular aortic stenosis. Circulation 1994; 89: 642-50.

5. Leon MB, Kodali S, Williams M, Oz M, Smith C, Stewart A, et al. Transcatheter aortic valve replacement in patients with critical aortic stenosis: rationale, device descriptions, early clinical experiences, and perspectives. Semin Thorac Cardiovasc Surg 2006; 18: 165-74.

6. Chiam PT, Ruiz CE. Percutaneous transcatheter aortic valve implantation: Evolution of the technology. Am Heart J 2009; 157: 229-42.

7. Klein AA, Webb ST, Tsui S, Sudarshan C, Shapiro L, Densem C. Transcatheter aortic valve insertion: anaesthetic implications of emerging new technology. Br J Anaesth 2009; 103: 792-9.

8. Covello RD, Maj G, Landoni G, Maisano F, Michev I, Guarracino F, et al. Anesthetic management of percutaneous aortic valve implantation: focus on challenges encountered and proposed solutions. J Cardiothorac Vasc Anesth 2009; 23: 280-5.

9. Brown JM, Murtha W, Fraser J, Khoury V. Dynamic left ventricular outflow tract obstruction in critically ill patients. Crit Care Resusc 2002; 4: 170-2.

10. Billings FT 4th, Kodali SK, Shanewise JS. Transcatheter aortic valve implantation: anesthetic considerations. Anesth Analg 2009; 108: 1453-62.

11. Bauer F, Lemercier M, Zajarias A, Tron C, Eltchaninoff H, Cribier A. Immediate and long-term echocardiographic findings after transcatheter aortic valve implantation for the treatment of aortic stenosis: the Cribier-Edwards/Edwards-Sapien valve experience. J Am Soc Echocardiogr 2010; 23: 370-6. 